\title{
AVALIAÇÃO DO BEYOND BUDGETING COMO FERRAMENTA DE PLANEJAMENTO E CONTROLE NA VISÃo DOS GESTORES DE IES
}

\section{RESUMO}

A necessidade de melhores práticas de gestão atinge todas as empresas que buscam continuidade e os estudos que procuram fornecer novos instrumentos de auxilio à gestão surgem à medida que o conhecimento avança. $\mathrm{O}$ Beyond Budgeting, por exemplo, foi um modelo apresentado como substituto ao orçamento tradicional. O estudo, de caráter exploratório, identificou a percepção dos gestores de IES localizadas na região Sul do Brasil quanto ao uso do orçamento. Por meio de questionários estruturados, foi verificado se as críticas feitas ao modelo de orçamento tradicional se aplicam às IES, bem como se os benefícios indicados pelos defensores de novas abordagens também se aplicam a essas instituições. Como resultado principal, identificouse que as críticas feitas ao orçamento tradicional não se aplicam às entidades estudadas, e que a opção pelo Beyond Budgeting não traria mudanças para as instituições, visto que grande parte dos benefícios propostos pela nova ferramenta já é empregada nas IES.

Palavras-chaves: Beyond Budgeting. Gestão. IES. Orçamento. Planejamento.

\section{ABSTRACT}

The need of best management practices reaches all the companies that come across for continuity and the studies that look for to supply new instruments to the management they appear as the knowledge moves forward, among them, Beyond Budgeting was presented as substitute to the traditional budget. The present study, of exploratory character, looked for to identify the IES managers'view located in the Brazil South area with relationship to the budget use. Through structured questionnaires, it was looked for to know if the critics done to the model of traditional budget they are applied in IES as well as if the benefits indicated by new approaches defenders are applied to these institutions. As main result, we identified traditional budget critics are not applied at studied entities, and the option for Beyond Budgeting would not bring changes for the institutions, because a great part of benefits proposed by the new tool is already applied in the institutions.

Keywords: Beyond Budgeting. Management. IES. Budget. Planning
Rony Petson Santana de Souza

Carlos Eduardo Facin

Lavarda 


\section{INTRODUÇÃO}

Nos últimos anos, nota-se que o Brasil passou por um considerável crescimento do mercado educacional, mais especificamente do mercado voltado ao ensino superior. Esse crescimento fez com que os competidores do setor buscassem cada vez mais uma melhor gestão para os seus empreendimentos, quer sejam de grande ou pequeno porte. Essa gestão voltada para o controle e o desempenho poderia proporcionar uma maior segurança quanto à situação econômico-financeira no presente e nas suas projeções e perspectivas para o futuro.

Como artefato de planejamento e controle que poderia ser utilizado para proporcionar essa segurança, destaca-se o orçamento empresarial - uma das ferramentas de gestão mais utilizadas pelas instituições para controlar e acompanhar as metas estabelecidas e para avaliar o desempenho dos gestores.

Apesar de ser uma ferramenta de planejamento e controle bastante utilizada pelas empresas, as críticas quanto ao atual modelo orçamentário como ferramenta de medição de desempenho têm se intensificado conforme pesquisas de Hope e Fraser (2003a) e Pflaeging (2003). Os críticos dessa ferramenta afirmam que o tradicional modelo orçamentário é resultado de contratos inflexíveis de desempenho e limitam gestores a atingir metas rígidas e em curto prazo e que, diante de um cenário futuro dinâmico e incerto, torna-se uma ferramenta totalmente ineficaz.

Como opção para otimizar os processos de planejamento e controle de desempenho surgiu o Beyond Budgeting, desenvolvido na Europa no final da década de 90 por Beyond Budgeting Round Table (BBRT), fórum de empresas que discute os custos com o processo orçamentário tradicional, o que levou ao questionamento sobre sua necessidade e uma maneira alternativa de planejamento e gestão (BBRT, 2009).

Diante do exposto, surge o seguinte questionamento norteador desta pesquisa: Qual a visão dos gestores das IES em relação às propostas apresentadas pelo Beyond Budgeting ante o modelo de orçamento tradicional?

O objetivo principal desta pesquisa é, portanto, mostrar a percepção dos gestores das Instituições de
Ensino Superior - IES do Vale do Itajaí, microrregião de Blumenau, sobre as propostas do Beyond Budgeting em relação ao modelo orçamentário tradicional.

Nos últimos anos foram realizados no Brasil estudos sobre o Beyond Budgeting para avaliar o desempenho e a aplicabilidade da ferramenta. Contudo, nos trabalhosjárealizados, foram estudadas instituições comerciais, financeiras e industriais, como se pode ver nos trabalhos de Soares e Neves (2004), Barbosa e Parisi (2004), Max (2005), Cordeiro Filho (2007) e Peleias et al. (2008). O estudo da aplicabilidade desse novo artefato não foi, pois, verificado em instituições de ensino superior (IES), o que torna relevante a presente pesquisa ao estudar um tema ainda pouco discutido no Brasil, além de discorrer sobre um método que propõe a eliminação completa de um modelo de planejamento e controle muito utilizado em empresas brasileiras. Esta investigação traz também reflexões sobre a necessidade ou não da aplicação desse novo método em IES, as quais possuem características de gestão e de planejamento diferentes de empresas industriais, comerciais e financeiras. Nesse sentido, a pesquisa busca contribuir para o entendimento e a aplicabilidade desse novo método de planejamento e controle que está ganhando espaço em empresas no exterior.

O estudo está assim organizado: além desta primeira seção, de caráter introdutório, a seção 2 aborda os conceitos do Beyond Budgeting, dando enfoque à sua origem e às suas características, e o modelo orçamentário tradicional. com ênfase nas críticas aos defensores do Beyond Budgeting. Na seção 3 são apresentados os procedimentos metodológicos utilizados, a população e a amostra desta pesquisa. A seção 4 traz a análise dos resultados da visão dos gestores das IES do Vale do Itajaí, microrregião de Blumenau, sobre a proposta do Beyond Budgeting. Por fim, na seção 5 , são apresentadas as considerações finais com recomendações para futuras pesquisas, tendo em vista as limitações do estudo atual.

2 ORÇAMENTO TRADICIONAL E BEYOND BUDGETING 
Com base na premissa de que no atual cenário econômico, que se apresenta cada vez mais dinâmico e incerto, o orçamento tradicional, com suas metas inflexíveis, torna-se uma ferramenta de planejamento organizacional e/ou gestão de desempenho bastante contestável para os padrões atuais, e, portanto, muito criticada (PFLAEGING, 2003).

Para Pflaeging (2003), as mudanças na economia ao longo das últimas décadas fizeram com que alguns procedimentos de gestão considerados como padrão fossem questionados:

Nossas economias e sociedades mudaram substancialmente, ao longo das últimas décadas. Mas os métodos e as práticas de gestão continuam sendo as mesmas. Práticas que por muito tempo consideramos padrão, hoje devem ser questionadas: negociação de metas, planos fixos, avaliação de pessoas, controles de horários, bonificações e incentivos, orçamentos e comparações "orçado versus realizado" todos amplamente difundidos, mas será que são adequados ainda?

Após uma discussão de um conjunto de empresas inglesas, no final da década de 90, que questionavam o modelo orçamentário tradicional com suas metas fixas, sua gestão centralizada e seu alto custo de implantação, foi criada uma organização chamada de Beyond Budgeting Round Table (BBRT), cuja função era desenvolver um modelo de gestão que substituísse o modelo de orçamento tradicional.
Hope e Fraser (2003) definem o modelo orçamentário tradicional como um instrumento que fixa metas anuais, recompensa seus gestores pelo alcance dessas metas prefixadas, centraliza a elaboração do orçamento e controla rigidamente o aporte de recursos.

ABBRT, após concluir que o modelo tradicional de orçamento não poderia se adaptar ao cenário dinâmico que se apresentava às empresas, desenvolveu o Beyond Budgeting (além do orçamento, em tradução livre) como modelo de gestão flexível, descentralizado, com a participação dos gerentes operacionais e "[...] capaz de realizar a transição da época industrial para a era da informação" (Cordeiro Filho, 2007).

Segundo Pflaeging (2003), a BBRT é uma rede que desenvolve e acompanha as empresas que estão implementando o modelo de gestão Beyond Budgeting, o qual seria o modelo de gerenciamento de desempenho para o século 21.

Tendo como principais diretores Jeremy Hope e Robin Fraser, a BBRT lançou o Beyond Budgeting fundamentado em 12 princípios, apresentados no Quadro 1. Segundo a BBRT (2009), seis desses princípios estão relacionados a processos flexíveis de gestão de desempenho e o restante está relacionado à liderança descentralizada no processo de elaboração orçamentária.

Ainda, a BBRT (2009) lista 6 ferramentas que

Quadro 1: Princípios do Beyond Budgeting PRINCÍPIOS DO BEYOND BUDGETING

\begin{tabular}{|c|c|}
\hline Processos Inflexíveis & Liderança Descentralizadora \\
\hline $\begin{array}{l}\text { Metas aspiracionais móveis, ao invés de metas anuais fixas; deve- } \\
\text { se bater na concorrência, e não no orçamento. }\end{array}$ & $\begin{array}{l}\text { Governança baseada em valores e limites claros, e não } \\
\text { em regras detalhadas e orçamentos. }\end{array}$ \\
\hline $\begin{array}{l}\text { Sistema de recompensas pelo sucesso obtido, e não pela } \\
\text { consecução de metas prefixadas e individuais. }\end{array}$ & $\begin{array}{l}\text { Desempenho direcionado à obtenção do sucesso da } \\
\text { equipe em relação ao mercado, e não ao atendimento de } \\
\text { metas internas e prefixadas. }\end{array}$ \\
\hline $\begin{array}{l}\text { Planejamento como processo contínuo, inclusivo e orientado } \\
\text { para a ação, e não como um evento anual. }\end{array}$ & $\begin{array}{l}\text { Liberdade de ação, de modo a capacitar os executivos } \\
\text { para agir (empowerment), em vez de exigir cumprimento } \\
\text { irrestrito dos planos. }\end{array}$ \\
\hline $\begin{array}{l}\text { Alocacão de recursos conforme a demanda, e não por meio de } \\
\text { programações orçamentárias anuais. }\end{array}$ & $\begin{array}{l}\text { Responsabilidade com base na criação de rede de } \\
\text { pequenas unidades responsáveis pelos resultados, em vez } \\
\text { de hierarquias centralizadoras. }\end{array}$ \\
\hline $\begin{array}{l}\text { Coordenação das interações intraorganizacionais de forma } \\
\text { dinâmica, por mecanismos de mercado, e não por meio de ciclos } \\
\text { anuais de planejamento. }\end{array}$ & $\begin{array}{l}\text { Foco nos clientes, de forma a obter melhoria nos } \\
\text { resultados dos clientes, em vez de atingir metas internas } \\
\text { previamente negociadas. }\end{array}$ \\
\hline $\begin{array}{l}\text { Controles baseados em indicadores chaves relativos de } \\
\text { desempenho, e não por meio de desvios em relação aos planos. }\end{array}$ & $\begin{array}{l}\text { Informacão aberta a todos (transparência), em vez de } \\
\text { torná-la seletiva, criando hierarquias de acesso a ela. }\end{array}$ \\
\hline
\end{tabular}


devem ser utilizadas junto com o Beyond Budgeting na substituição do orçamento tradicional, relacionadas abaixo:

a) Modelo de valor para o acionista como EVA (Economic Value Aided) e VBM (Value Based Management).

b) Benchmarking.

c) Balanced Scorecard.

d) ABM (Activity Based Management) - Gestão baseada em atividades.

e) CRM (Customer Relationship Management) Gestão de relacionamento com os clientes.

f) Sistemas de informações gerenciais e previsionais atualizados.

Verifica-se que os defensores do Beyond Budgeting o consideram não apenas uma nova ferramenta de gestão, mas um modelo de gestão em que os processos são flexíveis desde sua implementação até as decisões gerenciais, com responsabilidades divididas e avaliação de resultados voltada para a comparação de resultados externos, ao invés de internos.

Segundo Johnson e Kaplan (1987), o modelo de gestão de desempenho tradicional foi desenvolvido em 1920 como ferramenta para auxiliar os gestores a controlar custos e fluxo de caixa e, posteriormente, adaptando-se à evolução das organizações, passou a ter a função informativa aos gestores para realizar a condução do negócio.

Horngren et al. (2000, p. 125) definem orçamento como "expressão quantitativa de um plano de ação futuro da administração para um determinado período". Apesar do desenvolvimento citado, diversos autores como Hope e Fraser (2003a), Soares e Neves (2004) e Cordeiro Filho (2007) apontam críticas ao modelo orçamentário tradicional, tais como:

a) o orçamento é oneroso e consome muito tempo na sua elaboração;

b) na elaboração do orçamento, subjetivismos como palpites ou hipóteses insustentáveis são levados em consideração;

c) metas inflexíveis em um cenário imprevisível tornam o modelo de orçamento tradicional uma ferramenta sem valor; d) o orçamento não mede corretamente o desempenho dos gestores, pois não leva em conta o desempenho dos concorrentes;

e) o modelo orçamentário constitui uma barreira a mudanças, principalmente no que diz respeito à alocação de recursos;

f) o orçamento limita a ação do gestor por estabelecer um objetivo predeterminado, indicando onde ele pode e não pode chegar;

g) na elaboração do orçamento, apenas a alta administração participa, não dando espaço para quem realmente "faz acontecer".

Hansen, Otley e Stede (2003) apresentam críticas entre as quais podem ser citadas: a centralização nas decisões com comandos verticais; o foco na redução do custo, ao invés da criação de valor; os orçamentos que exclusivamente objetivam o resultado financeiro fazem com que o modelo orçamentário tradicional provoque uma incompatibilidade com as decisões operacionais e estratégicas que enfatizam metas não financeiras.

Iribarne (1995) e BBRT (2009), além de afirmar que metas inflexíveis podem ser um terror para os gestores, apontam algumas outras desvantagens do orçamento tradicional: a) a previsão de custos, despesas e receitas diante das constantes mudanças do mercado é comparada por alguns autores com a dança da chuva, "[...] cujos resultados aleatórios apenas repousam sobre as crenças sublimadas dos participantes" (BBRT, 2009); b) a sanção final do gerente limita suas ambições ao que ele é particularmente acessível; c) o impedimento de respostas rápidas pelas organizações diante de mudanças no mercado; d) a não ligação à estratégia da empresa; e) o reforço da cultura centralizadora e da dependência; f) a possibilidade de indução ao comportamento não ético.

Segundo Hope e Fraser (2003a), as empresas, apesar de resistirem a centralizações e controle, continuam a adotar o modelo de orçamento tradicional, que reforça todas essas características. Esses autores afirmam que só com o completo esquecimento desse modelo e a adoção do Beyond Budgeting é que as empresas teriam reais condições de possuir uma ferramenta capaz de planejar e avaliar desempenhos conforme o mercado exige. 


\section{METODOLOGIA DA PESQUISA}

A pesquisa tem caráter exploratório, e traz esclarecimentos sobre a abordagem do Beyond Budgeting, que ainda é pouco discutido no Brasil. Sobre a pesquisa exploratória, Vieira (2002) afirma que nela o pesquisador visa ter uma maior familiaridade com o problema em estudo. Gil (1998) relata que a pesquisa exploratória é útil quando há pouco conhecimento sobre o tema pesquisado, sendo difícil formular hipóteses precisas e operacionalizáveis.

Em relação à abordagem do problema, esta pesquisa é classificada como quantitativa e tem como objetivo fazer uma análise geral sobre o tema nas IES da amostra. $\mathrm{O}$ instrumento utilizado para a coleta de dados foi um questionário estruturado adaptado de Soares e Neves (2004), dividido em duas partes: a primeira parte compõe-se de sete questões fechadas, relacionadas ao perfil do respondente; a segunda parte constitui uma avaliação, medida em escala de Likert, de 18 questões relacionadas ao tema orçamento, para as quais os respondentes especificam seu nível de concordância sobre a temática com as afirmações: Concordo Totalmente (5), Concordo, Indiferente, Discordo e Discordo Totalmente (1). Os dados são apresentados em seus valores médios e desvio padrão, valendo-se dos recursos da estatística descritiva.

Esse questionário foi aplicado a gestores das Instituições de Ensino Superior - IES do Vale do Itajaí, microrregião de Blumenau, cuja população é composta por 14 IES instaladas nas cidades de Blumenau e Brusque. Para a definição da população, foi realizada uma pesquisa no sítio da Secretaria de Planejamento do Estado de Santa Catarina, onde foram identificadas as cidades que formam a microrregião de Blumenau no Vale do Itajaí. Na sequência, foi feita uma pesquisa no MEC para estabelecer que instituições de ensino superior localizadas nas cidades que fazem parte do Vale do Itajaí iriam receber o questionário, característico de uma pesquisa de levantamento.

$\mathrm{Da}$ população estabelecida, seis instituições pertencem a dois grupos, cujos orçamentos foram elaborados de forma centralizada, e uma das instituições pesquisadas no MEC, apesar de manter cadastro ativo, não mantém suas atividades de ensino, fazendo com que a população diminuísse para nove IES, tendo seis delas respondido o questionário.

Tanto Gil(1998)quantoBeuren(2006)reconhecem que os levantamentos apresentam limitações. Nesta pesquisa, as principais limitações foram: a) apenas as IES do Vale do Itajaí, microrregião de Blumenau, foram pesquisadas; b) a pesquisa foi realizada com um gestor de cada IES, portanto, foi uma pesquisa baseada na visão do entrevistado, e não na visão de toda a instituição.

\section{ANÁLISE DOS RESULTADOS}

Sobre o perfil dos gestores e das IES pesquisadas, foi verificado que entre eles há apenas um do gênero feminino. Há uma predominância de instituições sem fins lucrativos, existem as particulares e uma única instituição pública. Entre os gestores pesquisados, metade possui escolaridade superior a especialização e apenas um tem escolaridade igual ou superior a doutorado; dos que possuem escolaridade igual ou inferior a especialização, apenas um possui unicamente a graduação. A maioria dos gestores ocupa os cargos de gerente financeiro e de diretor das IES e possui, em sua maioria, a formação acadêmica em administração e ciências contábeis.

Foi constatado que a maior parte dos gestores tem experiência superior a 7 anos de atuação na função desempenhada na IES. Essas informações sobre o perfil dos gestores indicam que eles são profissionais com ampla experiência na atividade, possuem formação acadêmica adequada para ocupar cargos administrativos que possibilitem a sua participação ativa no processo de elaboração e controle do orçamento.

$\mathrm{Na}$ Tabela 1 são apresentadas as médias das avaliações dos gestores das IES do Vale do Itajaí, microrregião de Blumenau, em relação à eficiência do orçamento tradicional, bem como o desvio-padrão, que significa o desvio de cada resposta em relação à média de cada questão.

Pela tabela percebe-se que a satisfação dos gestores com o orçamento para o controle das despesas e realizações das receitas apresenta uma média de 4,17, o que indica que estão satisfeitos com o processo orçamentário tradicional, além de 
considerarem que ele é um processo formado com base em dados preanalisados, não levando em consideração "palpites".

Tabela 1 - Percepção dos gestores quanto à eficiência do orçamento tradicional

\begin{tabular}{l|c|c}
\hline \multicolumn{1}{c|}{ QUESTÕES } & Média & $\begin{array}{c}\text { Desvio- } \\
\text { padrão }\end{array}$ \\
\hline $\begin{array}{l}\text { O processo orçamentário atende a } \\
\text { necessidade da empresa no controle das } \\
\text { despesas e nas realizações das receitas. }\end{array}$ & 4,17 & 0,41 \\
\hline $\begin{array}{l}\text { O orçamento é um processo demorado e } \\
\text { cheio de "palpites". }\end{array}$ & 2,33 & 1,37 \\
\hline $\begin{array}{l}\text { Quem conhece Beyond Budgeting, } \\
\text { Advanced Budgeting ou outra forma de } \\
\text { orçamento. }\end{array}$ & 3,17 & 1,72 \\
\hline
\end{tabular}

Fonte: Dados da pesquisa.

Cordeiro Filho (2007, p. 3) afirma que quanto a questões relacionadas com o processo dos negócios "[...] os orçamentos, em sua elaboração, são baseados em hipóteses muitas vezes insustentáveis e em palpites, ou mera extrapolação de tendências".

No entanto, é possível perceber também que a maioria dos gestores desconhece o Beyond elaboração do orçamento tradicional, abordada nas pesquisas de Cordeiro Filho (2007) e Hope (2009).

Tabela 2: Percepção dos gestores quanto à centralização no orçamento tradicional

\begin{tabular}{l|c|c}
\hline \multicolumn{1}{c|}{ QUESTÕES } & Média & $\begin{array}{c}\text { Desvio- } \\
\text { Padrão }\end{array}$ \\
\hline $\begin{array}{l}\text { Há uma participação na elaboração do } \\
\text { orçamento pelos responsáveis pelos } \\
\text { setores. }\end{array}$ & 4,17 & 0,41 \\
\hline
\end{tabular}

Fonte: Dados da pesquisa.

O resultado obtido por essa tabela indica que a percepção dos gestores da IES pesquisadas são diferentes das observações dos estudos de Cordeiro Filho (2007) e Hope (2009), os quais afirmam que o processo de elaboração do orçamento tradicional é centralizado e não permite participação das pessoas responsáveis pela execução dele. Nesta pesquisa, identificou-se que os gestores participantes não consideram a elaboração do orçamento como um processo centralizado, ou seja, para eles - que apresentaram um índice de concordância de 4,17 -, há uma participação dos responsáveis pelos diversos setores da instituição nessa elaboração.
Budgeting, o Advanced

Budgeting ou outra forma de orçamento, o que pode influenciar na análise dos gestores com relação ao orçamento tradicional, visto que por causa desse desconhecimento eles avaliam apenas o modelo orçamentário já em uso na sua empresa. Esse resultado é compartilhado

Tabela 3: Percepção

\begin{tabular}{l|c|c}
\hline \multicolumn{1}{c|}{ QUESTÕES } & Média & $\begin{array}{c}\text { Desvio- } \\
\text { Padrão }\end{array}$ \\
\hline $\begin{array}{l}\text { Existe uma flexibilidade na liberação de recursos, caso o aporte financeiro } \\
\text { ultrapasse o que foi inicialmente orçado. }\end{array}$ & 3,17 & 0,98 \\
\hline $\begin{array}{l}\text { Na realização do orçamento leva-se em conta o desempenho da concorrência, e } \\
\text { não o resultado do exercício anterior. }\end{array}$ & 2,17 & 1,47 \\
\hline $\begin{array}{l}\text { Se no terceiro trimestre apenas 40\% das despesas orçadas forem realizadas, } \\
\text { os gestores passam a ser mais flexíveis com as solicitações de liberação de } \\
\text { recursos. }\end{array}$ & 2,83 & 1,72 \\
\hline Metas muito elevadas desestimulam a busca para alcançá-las. & 3,17 & 1,17 \\
\hline $\begin{array}{l}\text { Há um acompanhamento com intervalo de tempo no máximo trimestral para } \\
\text { avalizar o orçado versus o realizado. }\end{array}$ & 4,00 & 1,55 \\
\hline
\end{tabular}

Fonte: Dados da pesquisa. por Oyadomari et al. (2008, p. 8), que atestam que o Beyond Budgeting é o menos utilizado pois é o menos conhecido ou o mais contemporâneo, quando afirmam que “[...] quanto aos artefatos ligados às técnicas orçamentárias os mais contemporâneos possuem menor uso, e o Beyond Budgeting [...] mostrou-se o menos utilizado, o que confirma a utilização prioritária do orçamento nas empresas brasileiras".

$\mathrm{Na}$ Tabela 2 é demonstrada a percepção dos gestores das IES quanto à crítica da centralização na
Na Tabela 3 é apresentada a percepção dos gestores das IES sobre a característica flexibilidade do orçamento, em relação à qual Cordeiro Filho (2007, p. 3) faz uma crítica ao modelo orçamentário tradicional ao afirmar que quanto às estratégias competitivas “[...] os orçamentos, além de frequentemente constituírem barreiras às mudanças, restringem a responsividade $\mathrm{e}$ a flexibilidade".

Percebe-se que, diferentemente do que divulgam os críticos do orçamento tradicional, para os gestores 
das IES pesquisadas existe uma flexibilidade no orçamento, ou seja, o orçamento não são metas inflexíveis que engessam as ações deles, como afirma Pflaeging (2003). As discordâncias entre esses críticos e a percepção dos gestores das IES estudadas continuam quando o acompanhamento entre o orçado $\mathrm{x}$ o realizado é posto em questão. Para os críticos, o fato de o orçamento ser uma ferramenta anual e inflexível em meio a um cenário econômico bastante volátil torna-o uma ferramenta defasada a cada mudança do cenário econômico (Cordeiro, 2007).

Entretanto, para os gestores das aludidas IES, o orçamento tem um acompanhamento em um curto período de tempo, o que possibilita que o orçado seja adaptado à realidade atual da empresa e não se tenha de esperar o fim do exercício para refazê-lo por completo.

Em relação ao estabelecimento de metas elevadas - além da capacidade real - e o desestímulo proporcionado em alcançá-las, os gestores, em média, concordam que nessas condições os responsáveis pela execução delas se sentem desestimulados e podem pensar da seguinte maneira "já que essa meta é muito alta e não vamos alcançá-la, não adianta nem tentar" (PFLAEGING, 2003, p. 72XXXX).

$\mathrm{Na}$ tabela seguinte, foi

Tabela 5 - Percepção dos gestores quanto à utilização do orçamento como avaliação de desempenho

\begin{tabular}{l|c|c}
\hline \multicolumn{1}{c|}{ QUESTÕES } & Média & $\begin{array}{c}\text { Desvio- } \\
\text { Padrão }\end{array}$ \\
\hline É uma ferramenta útil na avaliação de desempenho dos gestores. & 4,00 & 1,10 \\
\hline $\begin{array}{l}\text { A análise do resultado orçamentário é suficiente para verificar o } \\
\text { desempenho da empresa. }\end{array}$ & 3,17 & 1,47 \\
\hline
\end{tabular}

Fonte: Dados da pesquisa.

Nota-se que praticamente todos os gestores analisam o resultado do orçamento para planejar as metas da empresa para o ano seguinte. Entretanto, identificouse nesta pesquisa que para a elaboração do orçamento nessas IES considera-se tanto questões externas, como os concorrentes, quanto à análise da oposição orçado versus realizado.

Esse resultado, em parte, contradiz os críticos do orçamento tradicional, quando afirmam que a adoção do Beyond Budgeting faria com que as empresas tivessem uma visão "para fora", ou seja, teriam seus planejamentos orçamentários com base no ambiente externo, como a concorrência ou a satisfação dos clientes (BBRT, 2009). As ações seriam mobilizadas por meio da visualização do mercado, e não pela busca interna para se alcançar metas que poderiam estar aquém das praticadas no mercado. Com a análise desses dados e da segunda questão da Tabela 3 , constata-se que nessas instituições o orçamento tradicional já aplica algumas das vantagens atribuídas ao Beyond Budgeting. analisada a percepção dos gestores quanto ao alinhamento do orçamento com o planejamento estratégico das IES.

$\mathrm{Na}$ Tabela 5 é apresentada a percepção dos gestores em relação ao uso do orçamento tradicional

Tabela 4: Percepção dos gestores quanto ao alinhamento do orçamento ao planejamento

\begin{tabular}{l|c|c}
\hline \multicolumn{1}{c|}{ QUESTÕES } & Média & $\begin{array}{c}\text { Desvio- } \\
\text { Padrão }\end{array}$ \\
\hline As metas estabelecidas no orçamento são alinhadas ao planejamento estratégico. & 3,83 & 0,75 \\
\hline O orçamento do exercício posterior é realizado com base na relação orçado x realizado. & 3,83 & 1,17 \\
\hline $\begin{array}{l}\text { Os resultados do orçamento são utilizados como uma forma de a empresa buscar uma } \\
\text { melhora para o próximo exercício. }\end{array}$ & 4,50 & 0,55 \\
\hline
\end{tabular}

Fonte: Dados da pesquisa.

Observa-se que a maioria dos gestores afirmou que a elaboração do orçamento é alinhado ao planejamento estratégico da empresa, com uma média de 4,50. como avaliação de desempenho. Cordeiro Filho (2007, p. 4) afirma que o orçamento, como ferramenta rígida que não permite alterações hábeis às mudanças no mercado, ao ser utilizado como referência para avaliação de desempenho dos gestores, pode provocar problemas como "[...] o desencorajamento dos indivíduos, em 
virtude de seus esforços reais serem ignorados e somente os resultados planejados serem levados em consideração".

Por essa tabela, depreende-se que, em média, os respondentes admitem que o orçamento é uma ferramenta útil para a avaliação do desempenho dos gestores. No entanto, quando indagados se apenas a análise do orçamento seria suficiente para verificar o desempenho da empresa, os gestores responderam menos positivamente, indicando que na visão deles há necessidade maior do uso de outro instrumento para medição do desempenho da empresa do que para a avaliação do desempenho deles. Pela análise da Tabela 5 , aliada à crítica ao orçamento tradicional - por utilizar ferramentas rígidas desencorajadoras da criatividade dos gestores, limitando-lhes as ações ao cumprimento das metas prefixadas (CARVALHO FILHO, 2007) e se tornando, portanto, uma ferramenta ineficaz para a avaliação dos gestores (BBRT, 2009) - pode-se inferir que, nas IES selecionadas, o orçamento (apesar da percepção dos gestores quanto à utilidade dele para a avaliação de desempenho) poderia ser utilizado em conjunto com outras medidas, o que evidentemente não significa afirmar que o planejamento orçamentário seja uma ferramenta ineficaz ou que não deva ser levada em consideração.

$\mathrm{Na}$ Tabela 6 é apresentada a percepção dos gestores das IES em questão quanto à utilização do orçamento tradicional como parâmetro para a atribuição de recompensas.

Tabela 6: Orçamento tradicional como parâmetro para recompensas

\begin{tabular}{l|c|c}
\hline \multicolumn{1}{c|}{ QUESTÕES } & Média & $\begin{array}{c}\text { Desvio- } \\
\text { Padrão }\end{array}$ \\
\hline Ao alcançar as metas orçamentárias, os gestores são gratificados. & 2,50 & 1,64 \\
\hline $\begin{array}{l}\text { As gratificações são proporcionais à capacidade de alcance das metas } \\
\text { orçamentárias. }\end{array}$ & 2,33 & 1,75 \\
\hline $\begin{array}{l}\text { Existe uma queda no ritmo quando as metas orçamentárias } \\
\text { relacionadas às receitas são alcançadas. }\end{array}$ & 2,50 & 1,38 \\
\hline Gratificações elevadas são estímulos para alcançar as metas. & 3,50 & 1,52 \\
\hline
\end{tabular}

Fonte: Dados da pesquisa.

Pela tabela acima, percebe-se que os gestores consideram que gratificações elevadas configuram estímulos para o alcance das metas. Deduz-se também que, em média, os gestores se mantiveram indiferentes em relação às afirmativas de que eles são gratificados quando alcançam as metas e que após as alcançarem diminuem o ritmo das suas atividades. Infere-se, ainda, que o único ponto de discordância entre os gestores foi quando se lhes perguntou se existia relação entre o valor das gratificações e o alcance das metas. Isto é, se no caso de alcance de $100 \%$ das metas haveria para eles ganho de toda a gratificação prometida, e se no caso de alcance das metas em percentagens inferiores às objetivadas haveria gratificações proporcionais.

\section{CONSIDERAÇÕES FINAIS}

O objetivo principal neste estudo foi verificar qual a percepção dos gestores das IES do Vale do Itajaí, microrregião de Blumenau, sobre as propostas do Beyond Budgeting em relação ao modelo orçamentário tradicional; já os objetivos específicos foram: a) verificar a percepção dos gestores das IES locais em relação ao Beyond Budgeting, e b) examinar a percepção dos gestores das IES locais em relação ao modelo de orçamento tradicional.

Com a aplicação de questionários, identificouse a avaliação dos gestores das IES sob análise no que diz respeito ao orçamento tradicional e ao Beyond Budgeting e pôde-se concluir que a crítica que se faz à eficácia do orçamento tradicional não é compartilhada unanimemente pelos gestores dessas IES. Os respondentes afirmaram positivamente que o orçamento atende à necessidade das empresas, atingindo uma média de 4,17 , entretanto não são todos os gestores que conhecem outra forma de orçamento, o que pode ser caracterizado como um viés à avaliação da qualidade do modelo orçamentário tradicional, mas que não permitiria inferir que o modelo é uma ferramenta ineficaz para as empresas investigadas.

Quanto às críticas ao modelo orçamentário tradicional em referência à centralização em sua elaboração, à sua inflexibilidade e à falta do seu alinhamento com o planejamento 
estratégico da empresa, não houve nenhuma concordância entre os críticos do orçamento e os gestores das IES sob referência. O único ponto comum entre eles apareceu quando os gestores, em média, concordaram que na elaboração do orçamento são levados em consideração os resultados do exercício anterior, e não os resultados da concorrência. Contudo, eles afirmaram que o orçamento é atualizado e reavaliado em um período máximo de três meses, o que poderia solucionar a questão de metas sub ou superavaliadas.

No que concerne à crítica da utilização do orçamento como avaliação de desempenho e como parâmetro para a recompensa dos gestores, mais uma vez não houve concordância entre os defensores do Beyond Budgeting críticos do orçamento tradicional e os gestores das IES estudadas. Sob o ponto de vista dos gestores, o orçamento é uma ferramenta útil para a avaliação de desempenho apesar de eles admitirem que o planejamento orçamentário deva ser utilizado concomitante a outras medidas de desempenho.

Pôde-se concluir que para os gestores a opção pelo Beyond Budgeting não traria grandes mudanças para as instituições, visto que grande parte dos benefícios propostos pela nova ferramenta já são aplicados nas instituições com o uso do modelo orçamentário tradicional, ou seja, as críticas a esse modelo não se aplicam às IES enfocadas.

Por fim, esta pesquisa identificou que as críticas feitas ao orçamento tradicional estão mais relacionadas com o mau uso do instrumento orçamentário, pois, se utilizado adequadamente, ele minimizaria os efeitos os quais, segundo alguns autores, só seriam de menor importância com o abandono por completo do orçamento tradicional. Portanto, na percepção dos gestores das IES em estudo não se constatou a necessidade do abandono total do orçamento tradicional.

\section{REFERÊNCIAS}

BBRT. Beyond Budgeting: Enabling the lean, adaptive and ethical enterprise. Disponível em: <http://www.bbrt. org>. Acesso em: 08 mai. 2009.

BARBOSA FILHO, F.; PARISI, C. A proposta do modelo beyond budgeting: modelo coerente de gestão sem ênfase em orçamentos. VII SEMEAD - FEA/USP. São Paulo, 2004. Anais ... FEA/USP, 2004. 1 CD ROM.

BARBOSA FILHO, Francisco. Estudo de caso de uma empresa brasileira de grande porte do ramo de alimentos, para analisar sua tendência ao modelo Beyond Budgeting. Dissertação de mestrado em Controladoria e Contabilidade Estratégica - Centro Universitário Álvares Penteado - UNIFECAP, São Paulo: 2004.

BEUREN, Ilse Maria; COLAUTO, Romualdo Douglas. Coleta, análise e interpretação dos dados. In: BEUREN, Ilse Maria (Org.). Como elaborar trabalhos monográficos em contabilidade. 3 ed., São Paulo: Atlas, 2006.

CORDEIRO FILHO, J. B. Orçamento como ferramenta de gestão: do orçamento tradicional ao Advanced Budgeting. XIV Congresso Brasileiro de Custos. João Pessoa, 2007. Anais ... 2007. CD- ROM.

FREZATTI, F. Além do orçamento: existiria alguma chance de melhoria do gerenciamento? Brazilian Business Review. Vitória, v.1, n.2, 2004, p.122-140.

GIL, Antonio Carlos. Metodologia do ensino superior. São Paulo: Atlas, 1998.

HANSEN, Stephen C.; OTLEY, David; STEDE, Win A.Van der. Practice developments in budgeting: an overview and research perspective. Journal of Management Accounting Research. Jan 2003.

HORNGREN, Charles T.; FOSTER, George; DATAR, Srikant M. Contabilidade Gerencial. Rio de Janeiro: LTC, 2000.

HOPE, J.; FRASER, R. Beyond budgeting: how managers can break free from the annual performance trap. Massachusetts: Harvard, 2003a.

HOPE, Jeremy. Beyond Budgeting: breaking through the barrier to "the third wave". Management Accounting. Dez 2007. Disponível em: <http://www.beyondbudgeting.com>. Acesso em: 07 jul. 2009.

IRIBARNE, Patrick. Les Tableaux de Bord de la performance. Paris: DUNOD, 1995.

JOHNSON, H. Thomas; KAPLAN, Robert S. Relevance Lost: The Rise and Fall of Management Accounting. USA: Harvard Business School Press, 1987.

MAX, M. Beyond Budgeting: case studies in north american financial services. Journal of Performance Management, Mar 2005, vol. 18 Issue 1, p. 3-15, 13 p. 
OYADOMARI, J. C. T. et al. Fatores que influenciam a adoção de artefatos de controle gerencial nas empresas brasileiras: um estudo exploratório sob a ótica da teoria neoinstitucional. In: Congresso USP, ..2008. Anais... São Paulo: USP, 2008 - CD-ROM.

PFLAEGING, Niels. Beyond Budgeting: como gerenciar o desempenhoparaobterummelhorresultado.In:SEMINÁRIO INTERNATIONAL BUSINESS COMMUNICATIONS (IBC), 2003. Anais... São Paulo, 2003.

PELEIAS, Ivam Ricardo, et al. Beyond Budgeting Percepções e Adaptabilidade ao Varejo Bancário Brasileiro: um estudo de caso. In: Congresso Brasileiro de Custos, 15, 2008, Curitiba. Anais... Curitiba: CBC, 2008. CD-ROM.

VIEIRA, Valter Afonso. As tipologias, variações e características da pesquisa de marketing. Revista FAE, Curitiba, v. 5, n. 1, p. 61-70, jan/abr. 2002.

SOARES, O. J.; NEVES JÚNIOR, I. José. Beyond Budgeting: Como otimizar o processo de gestão sem orçamentos. In: Congresso USP, ..2004. Anais...São Paulo: USP, 2004 - CD-ROM. 